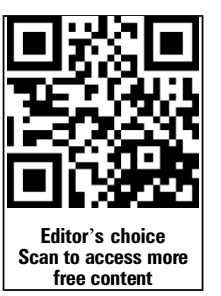

free content

\title{
Pittsburgh Response to Endovascular therapy (PRE) score: optimizing patient selection for endovascular therapy for large vessel occlusion strokes
}

\author{
Srikant Rangaraju, ${ }^{1}$ Amin Aghaebrahim, ${ }^{2}$ Christopher Streib, ${ }^{2}$ Chung-Huan Sun, \\ Marc Ribo, ${ }^{3}$ Marion Muchada, ${ }^{3}$ Raul Nogueira, ${ }^{1}$ Michael Frankel, ${ }^{1}$ Rishi Gupta, ${ }^{4}$ \\ Ashutosh Jadhav, ${ }^{2}$ Tudor $\mathrm{G}$ Jovin $^{2}$
}

- Additional material is published online only. To view please visit the journal online (http://dx.doi.org/10.1136/ neurintsurg-2014-011351).

${ }^{1}$ Marcus Stroke \& Neuroscience Center, Grady Memorial Hospital, Emory University, Atlanta, Georgia, USA ${ }^{2}$ Department of Neurology, University of Pittsburgh Medical Center, Pittsburgh, Pennsylvania, USA ${ }^{3}$ Unitat d'Ictus Vall d'Hebron Neurology Hospital Vall d'Hebron, Barcelona, Spain ${ }^{4}$ Wellstar Neurosurgery, Atlanta, Georgia, USA

Correspondence to Dr Tudor G Jovin, Stroke Institute, 200 Lorthop Street, Pittsburgh, PA 15213, USA; jovitg@upmc.edu

Received 25 June 2014 Revised 1 September 2014 Accepted 4 September 2014 Published Online First 15 October 2014

\section{CrossMark}

To cite: Rangaraju $S$, Aghaebrahim A, Streib C, et al. J Neurolntervent Surg 2015;7:783-788.

\begin{abstract}
Background Endovascular therapy seems to benefit a subset of patients with large vessel occlusion strokes. We aimed to develop a clinically useful tool to identify patients who are likely to benefit from endovascular therapy.

Methods In a derivation cohort of consecutively treated patients with anterior circulation large vessel occlusion (Grady Memorial Hospital, N=247), independent predictors $(p<0.1)$ of good outcome (90-day modified Rankin scale score (mRS) 0-2) were determined using logistic regression to derive the Pittsburgh Response to Endovascular therapy (PRE) score as a predictor of good outcome. The PRE score was validated in two institutional cohorts (University of Pittsburgh Medical Center (UPMC): N=393; Unitat $d^{\prime}$ Ictus Vall d'Hebron: $\mathrm{N}=204$ ) and its discriminative power for good outcome was compared with other validated tools. Benefit of successful recanalization was assessed in PRE score groups.
\end{abstract}

Results Independent predictors of good outcome in the derivation cohort (age, baseline National Institute of Health Stroke Scale (NIHSS) score and Alberta Stroke Program Early CT Score (ASPECTS)) were used in the model: PRE score $=$ age (years) $+2 \times$ NIHSS $-10 \times$ ASPECTS. PRE score was highly predictive of good outcome in the derivation cohort (area under the curve $(\mathrm{AUC})=0.79$ ) and validation cohorts (UPMC: AUC $=0.79$; UIVH: $A U C=0.72$ ) with comparable rates of good outcome in all PRE risk quartiles. PRE was superior to Totaled Health Risks In Vascular Events (THRIVE) ( $p=0.03$ ) and Stroke Prognostication using Age and NIHSS (SPAN) ( $p=0.007)$, with a trend towards superiority to Houston Intra-Arterial Therapy 2 (HIAT2) $(p=0.06)$ and iSCORE $(p=0.051)$ in predicting good outcomes. Better outcomes were associated with successful recanalization in patients with PRE scores -24 to +49 but not in patients with PRE scores $<-24$ or $\geq 50$.

Conclusions The PRE score is a validated tool that predicts outcomes and may facilitate patient selection for endovascular therapy in anterior circulation large vessel occlusions.

\section{INTRODUCTION}

Results from clinical trials comparing endovascular revascularization therapy (ERT) with intravenous thrombolysis for anterior circulation large vessel occlusion stroke (LVOS) have yielded neutral results, ${ }^{1-3}$ but several lines of evidence suggest that patients with severe stroke symptoms (National Institute of Health Stroke Scale (NIHSS) score $\geq 14$ ) and/or proximal LVOS (intracranial internal carotid terminus (ICA-T) and M1 middle cerebral artery (MCA)) may benefit from ERT. ${ }^{2}{ }^{45}$ In addition to the level of occlusion and NIHSS at presentation, several other factors (successful recanalization, established infarct core, Alberta Stroke Program Early CT Score (ASPECTS), age, baseline functional status and collateral status) also influence stroke outcome and need to be considered during patient selection for ERT. ${ }^{6}$ As a result, several prediction tools have been developed to aid patient selection, both in clinical practice as well as in defining the target population for clinical trials, ${ }^{7-10}$ but a standardized approach to patient selection for ERT is lacking across institutions. ${ }^{11}$ Optimal patient selection for ERT can minimize futile recanalization procedures, thereby limiting healthcare costs associated with ERT. ${ }^{12}$

Using independent predictors of good outcome that are available to the clinician prior to ERT, we derived the Pittsburgh Response to Endovascular therapy (PRE) score that incorporates age, NIHSS at presentation and ASPECTS on initial head CT scan and validated the PRE score in two large endovascular cohorts. The PRE score was also compared with previously validated scores that predict outcomes in patients with stroke. ${ }^{7}{ }^{13} 14$ PRE score thresholds were defined to distinguish patients likely to benefit from those who do not benefit from successful recanalization.

\section{METHODS}

Data source and subjects

The derivation cohort from the Grady Memorial Hospital (GMH) endovascular database (20082013) comprised consecutively treated patients. Demographic data, clinical data and 90-day modified Rankin scale score (mRS) as measured by the treating physician were prospectively collected for each patient. Adult patients (age $\geq 18$ years) with anterior circulation LVOS (ICA-T, M1 and M2 MCA) who received ERT within $8 \mathrm{~h}$ from time last-seen-well (TLSW) with documented 90-day mRS were included. The first validation cohort of consecutively treated patients (2009-2013) was derived from the University of Pittsburgh Medical 
Center (UPMC) endovascular stroke registry (Pittsburgh, Pennsylvania, USA). Patients in this cohort underwent endovascular therapy at one of three UPMC-affiliated hospitals, each a comprehensive stroke center with endovascular, neurointensive and rehabilitative services. The second validation cohort of consecutively treated patients (2009-2013) was derived from Unitat d'Ictus Vall d'Hebron Neurology Hospital Vall d'Hebron, Barcelona, Spain (UIVH). Each site had institutional review board approval for the maintenance of endovascular stroke databases. Permission from all three centers was sought prior to use of individual databases.

\section{Measurements}

Baseline NIHSS scores at each site were calculated by the treating physicians. ASPECTS on non-contrast head CT scan upon arrival at the comprehensive stroke center was determined by the treating neurologist or stroke physician prior to endovascular treatment. All patients underwent CT or MR angiography prior to endovascular treatment but level of occlusion was confirmed by catheter-based angiography. Revascularization status (Modified Thrombolysis In Cerebral Infarction (mTICI) grade) was determined by the operating physician after the procedure. Comparison of PRE score with Houston Intra-Arterial Therapy 2 (HIAT2), Totaled Health Risks In Vascular Events (THRIVE) and Stroke Prognostication using Age and NIHSS (SPAN) index was performed after pooling all three patient cohorts and excluding patients with missing data points. The HIAT2 score, THRIVE, iSCORE and SPAN index were calculated as previously described. ${ }^{7} 91014$ Final infarct volume $(\mathrm{mL})$ was determined by measuring the area of the infarct on each slice (diffusion-weighted MRI scan within the first $48 \mathrm{~h}$ following treatment or first available non-contrast head CT scan after $24 \mathrm{~h}$ of treatment) and then summating individual slice thicknesses of all outlined areas. ${ }^{15}$

\section{Statistical analysis}

Categorical variables were compared with the Fisher exact test. Continuous variables were compared using the unpaired t test (two-tailed) for means and independent samples median test for medians. Variables significantly predictive $(p<0.05)$ of good outcome (90-day mRS $0-2$ ) in univariate logistic regression analysis were entered in multivariate logistic regression analysis and only significant independent predictors of good outcome $(p \leq 0.1)$ were considered in the final model. Variables were weighted based on B coefficients relative to age (lowest B coefficient value). Each weight was rounded to the nearest integer and used in developing a composite score. Model calibration was assessed in the derivation cohort with the Hosmer-Lemeshow test ( $p>0.05$ considered good calibration). In univariate regression (derivation cohort) with PRE score as a predictor of good outcome, we determined the predicted rates of good outcome in different POST score groups and compared these with observed rates (Pearson correlation coefficient was determined). Receiver Operating Characteristic (ROC) area under the curve (AUC) was used to assess the discriminative power of PRE for good outcome (excellent $\geq 0.80$, very good $\geq 0.75$ ). Statistical analyses were performed using IBM SPSS Statistics V.20. The discriminative power (AUC) of PRE was compared with other scores using the Hanley and McNeil method. ${ }^{16}$

\section{RESULTS \\ Patient characteristics}

In the derivation cohort, 309 patients met the inclusion criteria and 62 patients were excluded due to missing imaging data or 90-day mRS. In the UPMC validation cohort, 426 patients met the inclusion criteria and 33 patients were excluded due to missing data variables. In the UIVH validation cohort, 244 patients met the inclusion criteria and 40 patients were excluded due to missing data variables. Patient characteristics and outcomes in the derivation $(\mathrm{GMH}, \mathrm{N}=247)$ and validation cohorts (UPMC, $\mathrm{N}=393$; UIVH, $\mathrm{N}=204$ ) are summarized in table 1. Significant differences in age, NIHSS, ASPECTS, sex distribution, risk factors (diabetes, hypertension and atrial fibrillation), proportion of ICA-T occlusions, intravenous thrombolysis rates and final infarct volumes were observed (table 1). In all three

Table 1 Patient characteristics in the derivation and validation cohorts

\begin{tabular}{|c|c|c|c|c|}
\hline Patient characteristics & $\begin{array}{l}\text { GMH (derivation) } \\
\mathrm{N}=247\end{array}$ & $\begin{array}{l}\text { UPMC (validation 1) } \\
\mathrm{N}=393\end{array}$ & $\begin{array}{l}\text { UIVH (validation 2) } \\
\mathrm{N}=204\end{array}$ & $\mathrm{p}$ Value \\
\hline Age, mean \pm SEM & $65.7 \pm 0.9$ & $66.4 \pm 0.7$ & $71 \pm 1$ & $<0.001$ \\
\hline Sex, N (\% male) & $140(52.8)$ & $187(47.6)$ & $113(55.4)$ & 0.045 \\
\hline NIHSS, median (IQR) & $18(15-23)$ & $16(12-20)$ & $19(17-21)$ & $<0.001$ \\
\hline ASPECTS, median (IQR) & $8(7-9)$ & $9(7-10)$ & $9(9-10)$ & $<.001$ \\
\hline $\mathrm{DM}, \mathrm{N}(\%)$ & $67(27.0)$ & $85(21.6)$ & $36(17.7)$ & 0.03 \\
\hline HTN, N (\%) & $180(72.6)$ & $256(65.1)$ & $132(64.7)$ & 0.04 \\
\hline Atrial fibrillation, $\mathrm{N}(\%)$ & $80(32.3)$ & $127(32.4)$ & $90(44.1)$ & 0.02 \\
\hline ICA, N (\%) & $58(23.5)$ & $100(25.5)$ & $74(36.3)$ & 0.004 \\
\hline M1 MCA, N (\%) & $158(63.9)$ & $249(63.4)$ & $116(56.9)$ & 0.22 \\
\hline M2 MCA, N (\%) & $31(12.6)$ & $44(11.1)$ & $14(6.9)$ & 0.13 \\
\hline TLSW to groin puncture (min), mean \pm SEM & $324 \pm 10.6$ & $336.4 \pm 32$ & $225 \pm 7$ & $<0.001$ \\
\hline IV tPA, N (\%) & $132(53.4)$ & $171(43.5)$ & $119(58.3)$ & 0.001 \\
\hline sICH rate, $\mathrm{N}(\%)$ & $18(7.3)$ & $25(6.4)$ & $19(9.3)$ & 0.4 \\
\hline Infarct volume (mL), median (IQR) & $38(17-89)$ & $64(22-164)$ & $35(8-150)$ & 0.001 \\
\hline 90-day mRS, median (IQR) & $3(2-6)$ & $4(2-6)$ & $4(1-6)$ & 0.28 \\
\hline
\end{tabular}

$\chi^{2}$ analysis was used to compare rates of occurrence of categorical variables. Age, TLSW to groin were compared using the Kruskal-Wallis test. Medians (for NIHSS, ASPECTS, infarct volume, $\mathrm{mRS}$ at 90 days) were compared using the Independent Samples Median test. $\mathrm{p}<0.05$ was considered significant.

ASPECTS, Alberta Stroke Program Early CT Score; DM, diabetes mellitus; GMH, Grady Memorial Hospital; HTN, hypertension; ICA, internal carotid artery; IV, intravenous; MCA, middle cerebral artery; mRS, modified Rankin scale score; NIHSS, National Institute of Health Stroke Scale; sICH, symptomatic intracerebral hemorrhage; tPA, tissue plasminogen activator;

TLSW, time from last-seen-well; UMPC, University of Pittsburgh Medical Center. 
cohorts the overall rate of good outcome was 40.1\% (339/844) regardless of recanalization status.

\section{Derivation and validation of the PRE score}

Univariate analysis in the derivation cohort identified age $(\mathrm{p}<0.001)$, NIHSS at admission $(\mathrm{p}<0.001), \quad$ ASPECTS $(p<0.001)$, female gender $(p=0.016)$, hypertension $(p=0.025)$, admission glucose level $(\mathrm{p}=0.02)$ and TLSW to groin puncture $(p=0.048)$ as significant predictors of good outcome $(p<0.05)$. Intravenous tissue plasminogen activator ( $\mathrm{tPA})(\mathrm{p}=0.8)$, level of occlusion $(p=0.26)$, hyperlipidemia $(p=0.09)$ and atrial fibrillation $(\mathrm{p}=0.87)$ were not significant predictors in this analysis and were excluded from the multivariate analysis. In a multivariate analysis (table 2), only age (OR $0.96,95 \%$ CI 0.94 to $0.99, \mathrm{p}=0.002$ ), NIHSS at presentation (OR $0.91,95 \%$ CI 0.86 to $0.97, \mathrm{p}=0.002$ ) and ASPECTS (OR 1.36, 95\% CI 1.21 to $1.51, \mathrm{p}=0.001$ ) on initial CT scan were independent predictors $(p<0.10)$ of good outcome. Calibration of the regression model was satisfactory in the derivation cohort (Hosmer-Lemeshow test $\mathrm{p}=0.36$ ). Individual factors were weighted based on B coefficients for each variable and a composite score was derived: PRE score $=$ age $+2 \times$ NIHSS $-10 \times$ ASPECTS. A very strong positive correlation was observed between predicted and observed rates of good outcome in the derivation cohort (figure 1A). Discriminative power of the PRE score was assessed by calculating the AUC of a ROC curve to predict a good outcome and was compared across PRE score groups in the derivation and validation cohorts. Compared with the derivation cohort (AUC $=0.79, \mathrm{p}<0.001$ ), PRE score predicted good outcomes in the UPMC (AUC 0.79, $\mathrm{p}<0.001$ ) and UIVH (AUC 0.72, $\mathrm{p}=0.001$ ) cohorts. Discriminative power of the PRE score was not affected by intravenous (IV) tPA use (AUC 0.794 (IV tPA) vs AUC 0.798 (non-IV tPA)). Patients were divided into PRE score quartiles (PRE $<0,0-24,25-49, \geq 50$ ) and predicted rates of good outcome were compared with observed rates in the derivation cohort. Compared with the derivation cohort, the validation cohorts had similar rates of good outcomes in PRE $<0(p=0.15)$, $0-24(p=0.15)$ and $\geq 50(p=1.0)$ groups (figure 1B). The PRE $25-49$ group in the validation cohorts had a lower rate of good outcome compared with the derivation cohort $(\mathrm{p}=0.03)$.

Since octogenarians represent a significant proportion of LVOS, a uniform approach to treating these patients is also needed. Octogenarians accounted for $20.8 \%(175 / 843)$ of the entire cohort. Of the 175 octogenarians, only 24 had PRE $\geq 50$ where ERT seems to be futile. In octogenarian patients with favorable PRE scores (PRE 0-49), successful recanalization (mTICI 2B/3) resulted in better outcomes compared with TICI $0-2 \mathrm{~A}$ recanalization $(35.2 \%$ vs $15 \%, \mathrm{p}=0.008)$, and this difference was more pronounced in the PRE $<25$ group $(52 \%$ vs $17 \%, p=0.004$ ) (see online supplementary efigure 2 ).

\section{Comparison of PRE score with other tools that predict} outcomes in acute LVOS

In a combined analysis of the derivation and validation cohorts we compared the PRE score with previously published scores (THRIVE, HIAT2, SPAN and iSCORE) that predict outcomes in acute ischemic stroke. In 707 patients in the derivation and validation cohorts (figure 1C), PRE (AUC $=0.79,95 \%$ CI 0.74 to $0.85, \mathrm{p}<0.001)$ was better than THRIVE $(\mathrm{p}=0.025)$ and SPAN $(p=0.007)$. PRE score was not statistically superior to HIAT2 although a trend was observed (PRE AUC $=0.79$ vs HIAT2 AUC $=0.75, p=0.06$ ). In 263 patients in the UPMC database with parameters available to calculate the iSCORE (100 patients excluded due to missing data), PRE trended towards superiority over iSCORE in predicting good outcome $(p=0.051$, figure 1D).

\section{PRE score can be used to identify patients likely to benefit from ERT}

The benefit of ERT is mediated through recanalization of the occluded vessel, thereby limiting infarct volume growth. With new generation endovascular devices, successful recanalization after ERT is achieved in about $70 \%$ of cases. In a combined analysis of 562 patients in the three cohorts (282 patients excluded due to missing mTICI status or final infarct volume), successful recanalization (mTICI $2 \mathrm{~B} / 3$ ) was achieved in 383 patients $(68.2 \%)$. We have shown that a PRE score $\geq 50$ is associated with a very low likelihood of good clinical outcomes following ERT. A true prediction of the benefit of ERT can be made if we compare outcomes in patients with and without successful recanalization (mTICI 2B/3) across various PRE score groups. In our database, successful recanalization resulted in higher rates of good clinical outcomes compared with mTICI $0-2 \mathrm{~A}$ in PRE score groups -24 to -1 $(\mathrm{p}=0.002), 0-24(\mathrm{p}<0.001)$ and $25-49(\mathrm{p}=0.001)$ but not in PRE score groups $\leq-25(\mathrm{p}=0.55)$ and $\geq 50(\mathrm{p}=1.0)$ (figure $2 \mathrm{~A}$ ). Rates of good outcome paralleled significantly smaller mean infarct volumes in the favorable PRE groups (PRE -24 to +49 ) but not in the PRE $\leq-25$ and PRE $\geq 50$ groups (figure $2 \mathrm{~B}$ ). The PRE $\leq-25$ and PRE $\geq 50$ groups together accounted for $13.2 \%$ (74/562) of patients in the combined database.

\section{DISCUSSION}

Results from recent trials and other studies comparing ERT with intravenous thrombolysis for $\operatorname{LVOS}^{1}$ have shown that ERT is not the treatment of choice for all LVOS but seems most effective in

Table 2 Results from multivariate analysis: Derivation of the PRE score

\begin{tabular}{|c|c|c|c|c|c|c|}
\hline Variable & B & $p$ Value & OR & $95 \% \mathrm{Cl}$ & Weight & Rounded \\
\hline Age & -0.037 & 0.002 & 0.96 & 0.94 to 0.99 & 1.0 & 1 \\
\hline NIHSS & -0.094 & 0.002 & 0.91 & 0.86 to 0.97 & 2.5 & 2 \\
\hline ASPECTS & 0.31 & 0.001 & 1.36 & 1.21 to 1.50 & -8.4 & -10 \\
\hline $\operatorname{Sex}(F)$ & -0.349 & 0.273 & 0.71 & 0.38 to 1.32 & & \\
\hline HTN & -0.333 & 0.337 & 0.72 & 0.36 to 1.41 & & \\
\hline Glucose & -0.004 & 0.168 & 0.99 & 0.99 to 1.002 & & \\
\hline TLSW to groin puncture & -0.003 & 0.143 & 0.99 & 0.99 to 1.001 & & \\
\hline
\end{tabular}

In univariate analysis, age, National Institute of Health Stroke Scale (NIHSS) score at admission, Alberta Stroke Program Early CT Score (ASPECTS), sex (female), hypertension (HTN), glucose at admission, time (min) from last-seen-well (TLSW) to groin puncture were significant pre-angiographic predictors of good outcome ( $<<0.05)$. Multivariate regression was performed after including these factors to identify independent predictors of good outcome $(p<0.10)$. Significant independent predictors were weighted based on B coefficients relative to age and rounded to the nearest convenient number.

Bold indicates that the three variables Age, NIHSS and ASPECTS were the only 3 significant independent predictors. 
A

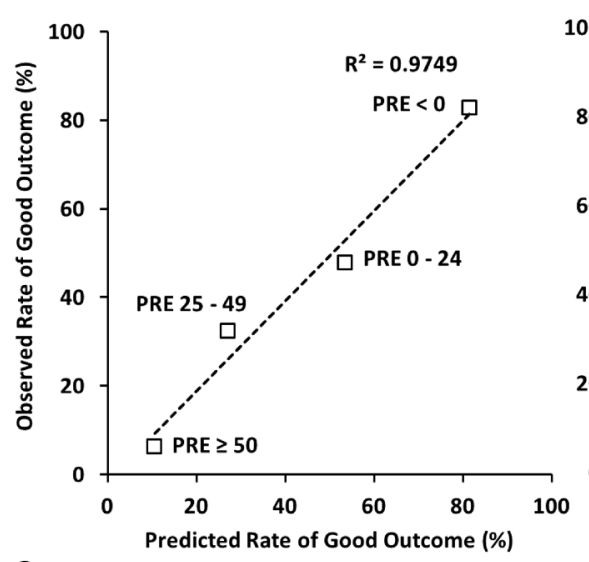

C

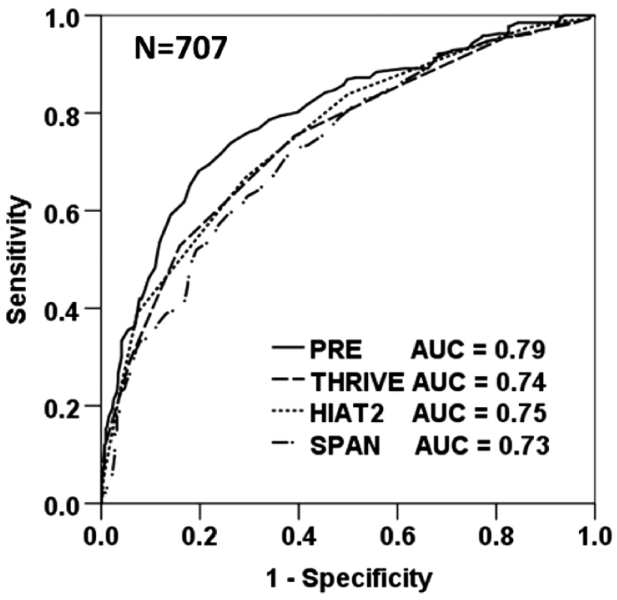

PRE Score $=$ Age $+2 \times$ NIHSS $-10 \times$ ASPECTS

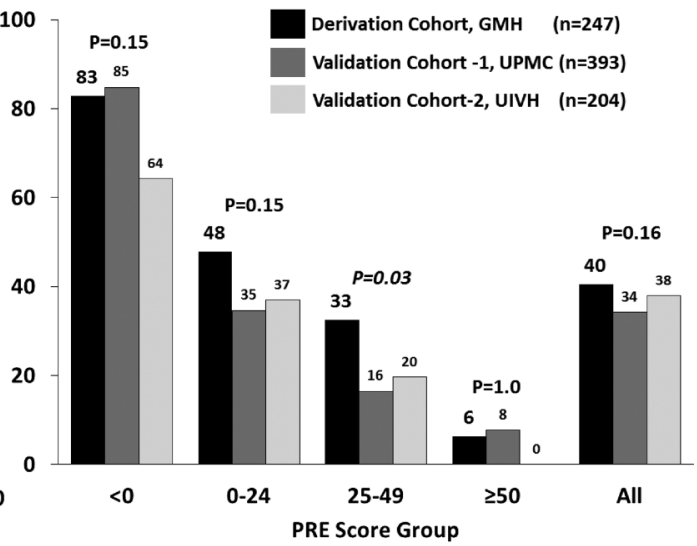

D

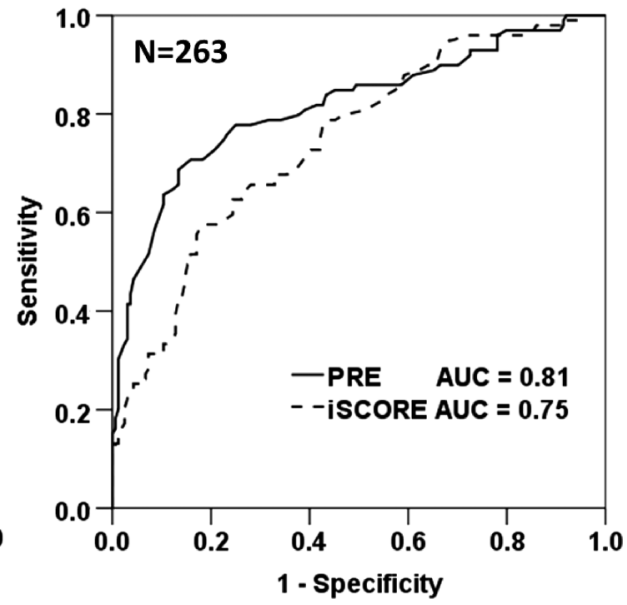

Figure 1 Pittsburgh Response to Endovascular (PRE) score validation and comparison with other validated clinical prediction scores. (A) Calibration of the PRE score in the derivation cohort: excellent degree of agreement between predicted and observed rates of good outcome across four PRE score groups $\left(R^{2}=0.98, p<0.001\right)$. (B) Comparison of the observed rates of good outcome in four PRE score groups in the derivation and validation cohorts showed no significant differences except for a slightly higher rate of good outcome in the PRE 25-49 group in the derivation cohort. (C, D) Comparison of discriminative power (area under the curve (AUC)) of PRE, THRIVE, HIAT2, iSCORE and SPAN for good outcomes (90-day modified Rankin scale score (mRS) 0-2). HIAT2, Houston Intra-Arterial Therapy 2; NIHSS, National Institute of Health Stroke Scale; SPAN, Stroke Prognostication using Age and NIHSS; THRIVE, Totaled Health Risks In Vascular Events.

proximal occlusions with higher NIHSS scores, ${ }^{2}$ that it is effective only if successful recanalization is achieved, ${ }^{3}$ and better patient selection is of key importance for future trials. ${ }^{4} 1718 \mathrm{It}$ is imperative to ensure that ERT is offered only to patients who are likely to benefit, while avoiding unnecessary interventions in patients who are either 'too good' or 'too bad' to treat. ${ }^{19}$ Several scoring tools for outcome prediction and patient selection for ERT have been developed but very few of these are routinely used in clinical practice. ${ }^{20}$

We developed and validated the PRE score to serve as a simple tool that can be used at the bedside to help clinicians in selecting patients with acute LVOS for ERT. The PRE score incorporates age, NIHSS at presentation and ASPECTS on initial CT scan as independent predictors of good outcome following ERT. This score is easy to calculate, incorporates factors that are commonly used in the acute setting before an MRI scan is available to determine the exact infarct burden and is independent of time to treatment or IV tPA use. Prognostication of good outcome prior to implementation of a potentially effective intervention should dichotomize the predictions based on success or failure of the intervention. The PRE score, when used in the context of revascularization status (mTICI $2 \mathrm{~B} / 3$ ), is able to identify subgroups of patients (PRE -24 to +49 ) who are likely to benefit from ERT (figure 2C). A PRE score $\geq 50$ identifies patients where ERT is very likely to be futile in preventing disability. This 'too bad to treat' population probably represents older patients with lower ASPECTS scores and higher NIHSS scores (see online supplementary eFigure 1). These results are also consistent with previous studies that identified age $>70$ and NIHSS score $\geq 20$ as independent predictors of futile recanalization in patients with LVOS. ${ }^{21}$ Patients with a PRE score $\leq-25$ seem to do well regardless of ERT, tend to be younger with lower NIHSS scores and higher ASPECTS (see online supplementary eFigure 1), but predictors of outcome in this population need to be defined. The ability to identify a 'too good to treat' population is a unique feature of the PRE score. In addition to being intuitive, the PRE score provides quantitative data to guide families or surrogate decision makers with regard to realistic expectations from endovascular interventions. A comparison of outcomes in successfully recanalized (mTICI $2 \mathrm{~B} / 3$ ) versus non-recanalized (mTICI $0-2 \mathrm{~A}$ ) patients also yields a rough estimate of the number of patients needed to be recanalized (NNR) in order to achieve one additional good outcome in each favorable PRE score group (PRE score -24 to 1: $\mathrm{NNR}=2.8 ; 0-24: \mathrm{NNR}=2.6 ; 25-49$ : $\mathrm{NNR}=3.85)$. While further validation of the PRE score in an endovascular trial 

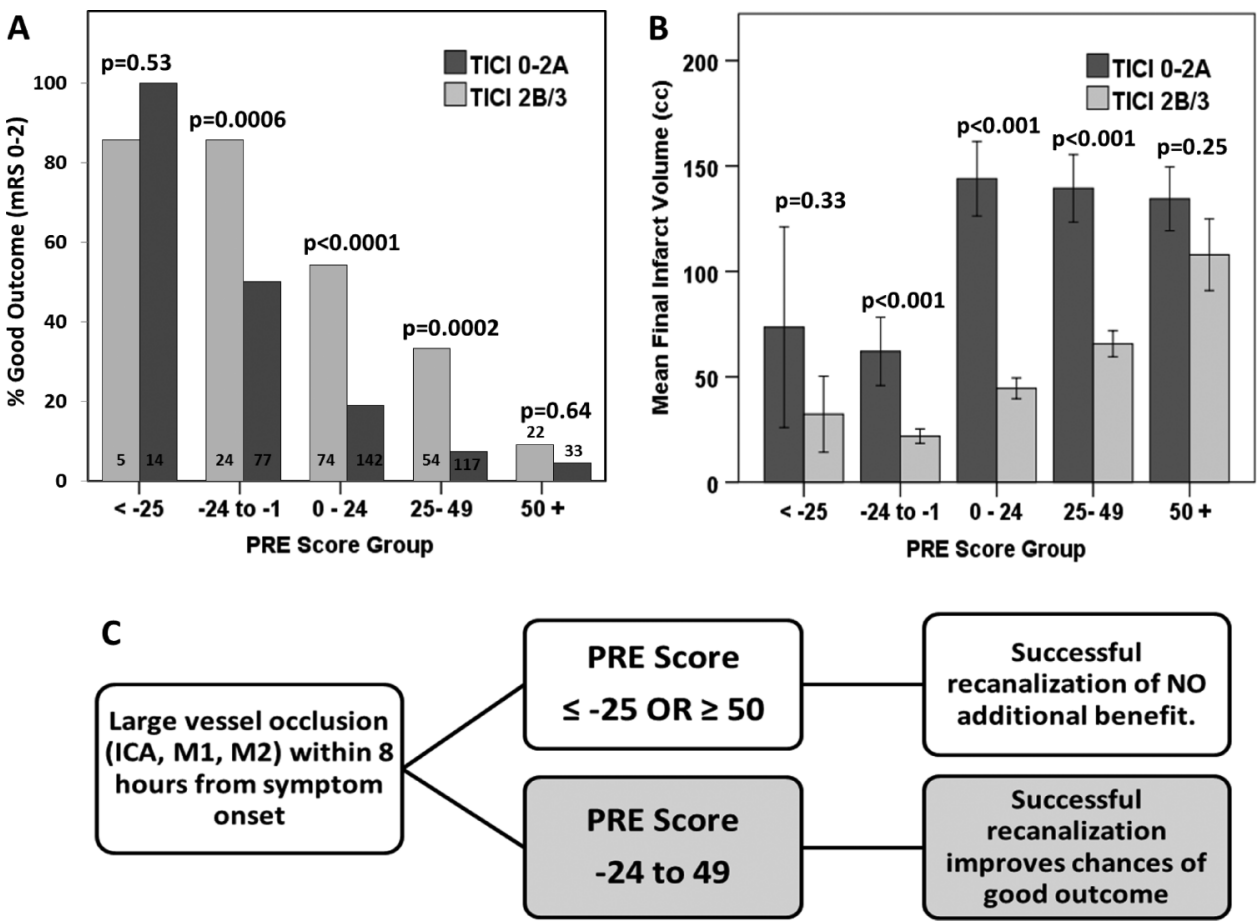

Figure 2 Identification of patients likely to benefit from recanalization with endovascular revascularization therapy (ERT). Comparison of (A) rates of good outcome (90-day modified Rankin scale score (mRS) 0-2) and (B) mean final infarct volumes in various Pittsburgh Response to Endovascular (PRE) score groups, dichotomized based on successful recanalization (Modified Thrombolysis In Cerebral Infarction (mTICl) 2B/3). (C) Suggested algorithm for using the PRE score in decision making for patient selection for ERT. ICA, internal carotid artery.

database would be useful in confirming our findings, these preliminary estimations may guide sample size calculations for future trials comparing ERT with intravenous thrombolysis within the $4.5 \mathrm{~h}$ time window as well as in trials enrolling patients beyond $4.5 \mathrm{~h}$ where tPA is not effective.

Clinical scoring systems are not meant to supersede clinical judgment but assist and build confidence in a medical decision made emergently, frequently by surrogate decision makers with guidance from the treating physician. Prognostic scores should also balance predictive accuracy with simplicity in order to be clinically useful. The SPAN index, for example, is a highly simplistic two-variable score with the lowest predictive accuracy in our analysis. The iSCORE, on the other hand, is a highly complex tool that incorporates numerous variables and cannot be determined without an online calculator. The THRIVE score, which incorporates age, NIHSS and three risk factors (diabetes mellitus, hypertension, atrial fibrillation), ${ }^{7}$ and the HIAT2 score, which incorporates age, NIHSS, glucose and ASPECTS, ${ }^{14}$ have both been validated in endovascular cohorts. Compared with these, the PRE score incorporates only three variables and, despite its simplicity, seems to be superior to THRIVE, HIAT2 and iSCORE. This could be due to use of age, NIHSS score and ASPECTS as continuous variables in our model rather than categorical variables, which could overcome any loss of predictive power resulting from categorization of variables. Whether a scoring tool such as the PRE score is helpful in clinical practice and decision making needs to be prospectively validated.

Studies have suggested that octogenarians with LVOS are less likely than younger patients to achieve successful recanalization or good outcomes following ERT. ${ }^{22}$ While our analysis in octogenarians is limited by small sample size and selection biases, we did find that patients with a favorable PRE score had better outcomes with successful recanalization, suggesting that ERT in octogenarians with PRE score $<50$ is reasonable and should be evaluated prospectively.

Hospital costs related to endovascular therapy can also be prohibitive in limited resource settings and a cost-effective analysis of ERT in acute ischemic stroke has not been performed. In our study, $13 \%$ of patients were very unlikely to benefit from ERT (PRE $\leq-25$ or PRE $\geq 50$ ). Excluding these patients from ERT may limit futile procedures and unnecessary hospital expenses at an aggressive endovascular center. Conversely, at a conservative center, procedure-related costs may increase due to a larger number of patients that can be considered for ERT, but this could be offset by lowered disability and nursing home or rehabilitation costs. Only results from ongoing clinical trials will confirm the benefit (or lack) of ERT in LVOS.

Certain limitations of our study need to be acknowledged. Our analysis is retrospective and is prone to selection bias due to varying endovascular practices at each institution. Significant differences in baseline demographics in our derivation and validation cohorts were also observed. Since patients without mRS at 90 days were excluded, selection bias due to patients lost to follow-up may explain the non-significant differences in median follow-up $\mathrm{mRS}$ in the derivation and validation cohorts. Nevertheless, the PRE score predicted clinical outcomes in a comparable manner, supporting its wide applicability to patients with anterior circulation LVOS regardless of epidemiologic disparities. Surprisingly, variables such as IV tPA use, level of occlusion and atrial fibrillation were not significant predictors of good outcome. This could be explained by the limited sample size of the derivation cohort and selection biases. It is also possible that, in the LVOS population, these variables have a smaller influence on outcome. Since all patients were treated with endovascular therapy, many patients may have been treated shortly after tPA administration, thereby possibly masking any potential recanalization-mediated benefit of IV tPA. Baseline disability was 
not included in our model due to the selection bias of not offering ERT to patients with significant baseline disabilities (mRS $\geq 3$ ) at most centers. Only $1 \%(2 / 204)$ patients who were treated at UIVH had a baseline $\mathrm{mRS} \geq 3$. The PRE score must therefore be applied with caution to patients with major baseline disabilities. Although the PRE score was not validated in a tPA-ineligible population, we expect comparable results since the discriminative power of PRE was not affected by the utilization of IV tPA. The PRE score was not validated in patients treated beyond $8 \mathrm{~h}$ from TLSW, but we expect the PRE score to retain its predictive accuracy in these patients because time to treatment was not an independent predictor of outcome in our analysis, an observation explained by the close association between time to treatment and ASPECTS. Finally, since the majority of patients were $>35$ years of age, the PRE score has not been validated in very young patients. Further validation of the PRE score in an endovascular trial database may overcome many of these limitations.

In conclusion, we have derived and validated the PRE score as a simple tool to predict outcomes in patients with LVOS prior to ERT. Patients with a PRE score of -24 to +49 seem to benefit from ERT if successful recanalization is achieved. The PRE score also performs better than previously validated scoring tools and can be useful in clinical practice and in future endovascular trials.

Contributors SR:study concept and design, acquisition of data, analysis and interpretation. AA, C-HS, MR, MM, AJ: acquisition of data, critical revision of manuscript for important intellectual content. CS, RN, RG, MF: critical revision of manuscript for important intellectual content. TGJ: study supervision, study concept and design, analysis and interpretation.

Competing interests RG: consultant to Stryker Neurovascular, Covidien, Rapid Medical, Royalties: UpToDate, Associate editor of Journal of Neuroimaging and Interventional Neurology. RN: Stryker Neurovascular (PI for TREVO-2 and DAWN Trials), Covidien (Steering Committee SWIFT trials; Core Lab-STAR trial), Penumbra (Executive Committee-3D Separator trial). TGJ: consulting and speaker fees from Co-Axia, ev3, Concentric Medical and Micrus.

Ethics approval Institutional Review Board (retrospective analysis).

Provenance and peer review Not commissioned; externally peer reviewed.

\section{REFERENCES}

1 Broderick JP, Palesch YY, Demchuk AM, et al. Endovascular therapy after intravenous t-PA versus t-PA alone for stroke. N Engl J Med 2013;368:893-903.

2 Singh B, Parsaik AK, Prokop $L$, et al. Endovascular therapy for acute ischemic stroke: a systematic review and meta-analysis. Mayo Clin Proc 2013;88:1056-65.
3 Chimowitz MI. Endovascular treatment for acute ischemic stroke-still unproven. N Engl J Med 2013;368:952-5.

4 Rangaraju S, Owada K, Noorian AR, et al. Comparison of final infarct volumes in patients who received endovascular therapy or intravenous thrombolysis for acute intracranial large-vessel occlusions. JAMA Neurol 2013;70:831-6.

5 Elliott KS, Chapman K, Day-Williams A, et al. Evaluation of the genetic overlap between osteoarthritis with body mass index and height using genome-wide association scan data. Ann Rheum Dis 2013;72:935-41.

6 Fields JD, Lutsep HL, Smith WS. Higher degrees of recanalization after mechanical thrombectomy for acute stroke are associated with improved outcome and decreased mortality: pooled analysis of the MERCI and Multi MERCI trials. AJNR Am J Neuroradiol 2011;32:2170-4.

7 Flint AC, Xiang B, Gupta R, et al. THRIVE score predicts outcomes with a third-generation endovascular stroke treatment device in the TREVO-2 trial. Stroke 2013;44:3370-5.

8 Ribo M, Flores A, Mansilla E, et al. Age-adjusted infarct volume threshold for good outcome after endovascular treatment. J Neurointerv Surg 2014;6:418-22.

9 Saposnik G, Guzik AK, Reeves M, et al. Stroke prognostication using age and NIH stroke scale: SPAN-100. Neurology 2013;80:21-8.

10 Saposnik G, Kapral MK, Liu Y, et al. IScore: a risk score to predict death early after hospitalization for an acute ischemic stroke. Circulation 2011;123:739-49.

11 Mehta B, Leslie-Mazwi TM, Chandra RV, et al. Assessing variability in neurointerventional practice patterns for acute ischemic stroke. J Neurointerv Surg 2013;5(Suppl 1):i52-7.

12 Molina CA. Futile recanalization in mechanical embolectomy trials: a call to improve selection of patients for revascularization. Stroke 2010;41:842-3.

13 Dzialowski I, Hill MD, Coutts SB, et al. Extent of early ischemic changes on computed tomography (CT) before thrombolysis: prognostic value of the Alberta Stroke Program Early CT Score in ECASS II. Stroke 2006;37:973-8.

14 Sarraj $A$, Albright $K$, Barreto $A D$, et al. Optimizing prediction scores for poor outcome after intra-arterial therapy in anterior circulation acute ischemic stroke. Stroke 2013;44:3324-30.

15 Vora NA, Shook SJ, Schumacher HC, et al. A 5-item scale to predict stroke outcome after cortical middle cerebral artery territory infarction: validation from results of the Diffusion and Perfusion Imaging Evaluation for Understanding Stroke Evolution (DEFUSE) Study. Stroke 2011;42:645-9.

16 Hanley JA, McNeil BJ. The meaning and use of the area under a receiver operating characteristic (ROC) curve. Radiology 1982;143:29-36.

17 Kidwell CS, Jahan R, Gornbein J, et al. A trial of imaging selection and endovascular treatment for ischemic stroke. N Engl J Med 2013;368:914-23.

18 Ciccone A, Valvassori L, Nichelatti $\mathrm{M}$, et al. Endovascular treatment for acute ischemic stroke. N Engl J Med 2013;368:904-13.

19 Yoo AJ, Leslie-Mazwi TM, Jovin TG. Future directions in IAT: better studies, better selection, better timing and better techniques. J Neurointerv Surg 2013;5(Suppl 1): i1-6.

20 Caplan LR. Scores of scores. JAMA Neurol 2013;70:252-3.

21 Hussein HM, Georgiadis AL, Vazquez G, et al. Occurrence and predictors of futile recanalization following endovascular treatment among patients with acute ischemic stroke: a multicenter study. AJNR Am J Neuroradiol 2010;31:454-8.

22 Duffis EJ, He W, Prestigiacomo CJ, et al. Endovascular treatment for acute ischemic stroke in octogenarians compared with younger patients: a meta-analysis. Int $\mathrm{J}$ Stroke 2013;9:308-12. 